\title{
Singularly Perturbed Spectral Problems in a Thin Cylinder with Fourier Conditions on its Bases
}

\author{
Andrey Piatnitski and Volodymyr Rybalko
}

The paper deals with the bottom of the spectrum of a singularly perturbed second order elliptic operator defined in a thin cylinder and having locally periodic coefficients in the longitudinal direction. We impose a homogeneous Neumann boundary condition on the lateral surface of the cylinder and a generic homogeneous Fourier condition at its bases. We then show that the asymptotic behavior of the principal eigenpair can be characterized in terms of the limit one-dimensional problem for the effective HamiltonJacobi equation with the effective boundary conditions. In order to construct boundary layer correctors we study a Steklov type spectral problem in a semi-infinite cylinder (these results are of independent interest). Under a structure assumption on the effective problem leading to localization (in certain sense) of eigenfunctions inside the cylinder we prove a two-term asymptotic formula for the first and higher order eigenvalues.

Key words: singularly perturbed operators, homogenization problems, eigenvalues, eigenfunctions, Fourier boundary conditions.

Mathematical Subject Classification 2010: 35B27,35P15, 35J25.

\section{Introduction}

In this work, we consider spectral problems for singularly perturbed second order elliptic operators defined in a thin cylinder with the Neumann boundary condition on the lateral surface of the cylinder and the Fourier boundary conditions on its bases. The scaling in the problem is such that these latter parts of the boundary create boundary layers which affect the overall behavior of the solutions and therefore require a thorough study.

Previously, spectral problems in a smooth bounded domain for a singularly perturbed second order elliptic operator with rapidly oscillating locally periodic coefficients were studied in $[11,12]$, where the case of Dirichlet boundary condition was studied. The methods used in these works rely essentially on homogenization techniques for (perturbed) Hamilton-Jacobi type equations with locally periodic coefficients, see, for instance, $[7,8]$. The effective boundary condition is the socalled state constraint boundary condition, more details can be found in $[5,14]$. 
When studying a similar problem with the Neumann or the Fourier boundary condition, we face a common in homogenization theory difficulty caused by the inconsistency of the periodic structure inside a domain with the boundary leading to very irregular boundary layers (see, e.g., [4]). However, in the case of a thin cylinder considered in the present work the boundary layers appearing near its bases can be successfully studied. To this end, we use a factorization argument to reduce the study of the mentioned boundary layers to the Steklov type spectral problems in a semi-infinite cylinder. Congenerous problems in half-space type domains (with periodic conditions instead of the Neumann condition on the lateral surface) were considered in [3] and [4]. The main novelty of the present work is the comprehensive description of the Steklov type spectral problems in the semi-infinite cylinder which includes uniqueness/non-uniqueness results in terms of the so-called effective longitudinal drift, and exhausting results in the case of non-uniqueness. These results, which are also of independent interest, allow us to identify effective boundary conditions. Note that the asymptotic analysis of the problem leads to the dimension reduction, and thus the effective HamiltonJacobi eigenvalue problem is one-dimensional. Due to this dimension reduction we are able to provide also the two-term asymptotic formulas for eigenvalues. Namely, under a structure assumption on the effective problem we reduce the original spectral problems to a form amenable to local asymptotic analysis on an intermediate scale. We prove the convergence (in norm) of resolvent operators to the resolvent operator of a one-dimensional harmonic oscillator problem. The eigenvalues of the latter problem provide the second term in the two-term asymptotic formulas mentioned above.

We are also to mention works $[1,2]$ close to the subject considered in the paper. The first work deals with the purely one-dimensional case, the second one describes an asymptotic behavior of the principal eigenfunction of the problem adjoint to a convection-diffusion problem in a thin cylinder with the Neumann condition on the boundary.

The paper is organized as follows. Section 2 describes the problem. In Section 3 , we study the principal eigenpair by means of the vanishing viscosity method. Section 4 is devoted to the construction of boundary layers near the bases of the cylinder and derivation of effective boundary conditions, it studies the Steklov type spectral problems in a semi-infinite cylinder. As already mentioned, the results of this Section might be of independent interest. In Section 5, we establish the two-term asymptotic formulas for the first and higher order eigenvalues under a structure assumption on the effective problem.

\section{Problem setup}

Given $L>0$, a smooth bounded domain $\omega \in \mathbb{R}^{n-1}$ and a small parameter $\varepsilon>0$, we consider an elliptic operator $\mathcal{L}_{\varepsilon}$ in the cylinder $(0, L) \times \varepsilon \omega$,

$$
\mathcal{L}_{\varepsilon} u=\varepsilon^{2} a_{i j}\left(x_{1}, x / \varepsilon\right) \frac{\partial^{2} u}{\partial x_{i} \partial x_{j}}+\varepsilon b_{j}\left(x_{1}, x / \varepsilon\right) \frac{\partial u}{\partial x_{j}}+c\left(x_{1}, x / \varepsilon\right) u .
$$


The coefficients $a_{i j}, b_{j}$ and $c$ are fast oscillating locally periodic functions: they depend on $x_{1}$ (slow variable) and $y=x / \varepsilon$ (fast variable) being 1-periodic in $y_{1}$. Since (2.1) contains the factor $\varepsilon^{2}$ in front of the higher order term and $\varepsilon$ in front of the first derivatives, $\mathcal{L}_{\varepsilon}$ is a singularly perturbed operator. We impose the homogeneous Neumann condition on the side boundary of the cylinder,

$$
\frac{\partial u}{\partial \nu_{a}}=0 \quad \text { on }(0, L) \times \varepsilon \partial \omega,
$$

where $\frac{\partial u}{\partial \nu_{a}}=a_{i j}\left(x_{1}, x / \varepsilon\right) \frac{\partial u}{\partial x_{j}} \nu_{i}$ is the conormal derivative with $\nu=\left(\nu_{1}, \ldots, \nu_{n}\right)$ being the unit normal vector (outward pointing), and consider the Fourier conditions

$$
\varepsilon \frac{\partial u}{\partial \nu_{a}}+g_{ \pm}\left(x^{\prime} / \varepsilon\right) u_{\varepsilon}=0, \quad \text { when } x=\left(x_{1}, x^{\prime} / \varepsilon\right) \in\left\{\frac{L \pm L}{2}\right\} \times \omega,
$$

on the bases of the cylinder.

Consider the spectral problem

$$
\mathcal{L}_{\varepsilon} u=\lambda u \quad \text { in }(0, L) \times \varepsilon \omega, u \text { satisfies }(2.2) \text { and (2.3). }
$$

Under some natural conditions (uniform ellipticity of the higher order term and smoothness of the coefficients) the spectrum of this problem is discrete. We study he asymptotic behavior of the eigenpairs as $\varepsilon \rightarrow 0$.

The exact conditions imposed on the coefficients of the operator $\mathcal{L}_{\varepsilon}$ are the following:

$a_{i j}\left(x_{1}, y\right), b_{j}\left(x_{1}, y\right), c\left(x_{1}, y\right) \in C^{3}([0, L] \times \mathbb{R} \times \bar{\omega})$ are 1-periodic

in $y_{1}$ functions, the symmetry $a_{i j}=a_{j i}$,

and the uniform ellipticity $a_{i j} \xi_{i} \xi_{j} \geq \gamma|\xi|^{2}>0\left(\forall \xi \in \mathbb{R}^{n} \backslash\{0\}\right)$ holds.

We also suppose that

$\omega$ is a bounded domain of the class $C^{2}$, and $g_{ \pm} \in C^{1}(\bar{\omega})$.

\section{Asymptotic behavior of the first eigenpair}

We begin by considering the first eigenvalue $\lambda_{\varepsilon}$ (with the maximal real part) of $\mathcal{L}_{\varepsilon}$. By the Krein-Routman theorem, $\lambda_{\varepsilon}$ is a simple eigenvalue and $\lambda_{\varepsilon} \in$ $\mathbb{R}$, the corresponding eigenfunction $u_{\varepsilon}$ can be chosen positive. As in the series of works [11-13], we represent the eigenfunction as $u_{\varepsilon}=e^{-W_{\varepsilon} / \varepsilon}$ arriving at a perturbed Hamilton-Jacobi equation

$$
-\varepsilon a_{i j}\left(x_{1}, x / \varepsilon\right) \frac{\partial^{2} W_{\varepsilon}}{\partial x_{i} \partial x_{j}}+H\left(x_{1}, x / \varepsilon, \nabla W_{\varepsilon}\right)=\lambda_{\varepsilon} \quad \text { in }(0, L) \times \varepsilon \omega,
$$

where $H\left(x_{1}, y, p\right)=a_{i j}\left(x_{1}, y\right) p_{i} p_{j}-b_{j}\left(x_{1}, y\right) p_{j}+c\left(x_{1}, y\right)$, subject to the boundary conditions:

$$
\frac{\partial W}{\partial \nu_{a}}=0 \quad \text { on }(0, L) \times \varepsilon \partial \omega, \frac{\partial W_{\varepsilon}}{\partial \nu_{a}}=g_{ \pm}\left(x^{\prime} / \varepsilon\right), \text { when } x_{1}=\frac{L \pm L}{2}, x^{\prime} \in \varepsilon \omega .
$$


It is not difficult to obtain a priori estimates ensuring that, up to a subsequence, $\lambda_{\varepsilon}$ converges to a finite limit $\lambda$, and $W_{\varepsilon}$ (normalized by $\min W_{\varepsilon}=0$ ) converges uniformly to a function $W\left(x_{1}\right)$. Moreover, one establishes (e.g., following the lines of [11]) that $W$ is a viscosity solution of the equation

$$
\bar{H}\left(x_{1}, W^{\prime}\right)=\lambda \quad \text { in }(0, L)
$$

with the effective Hamiltonian $\bar{H}\left(x_{1}, p_{1}\right)$ defined as the unique number such that the equation

$$
\begin{aligned}
a_{i j}\left(x_{1}, y\right) \frac{\partial^{2} \theta}{\partial y_{i} \partial y_{j}} & +\left(b^{j}\left(x_{1}, y\right)-2 a_{1 j}\left(x_{1}, y\right) p_{1}\right) \frac{\partial \theta}{\partial y_{j}} \\
& +H\left(x_{1}, y, p_{1}, 0, \ldots, 0\right) \theta=\bar{H}\left(x_{1}, p_{1}\right) \theta \quad \text { in } \mathbb{R} \times \omega
\end{aligned}
$$

has a positive solution $\theta=\theta\left(y, x_{1}, p_{1}\right)$ which is 1 -periodic in $y_{1}$ and satisfies the boundary condition

$$
\frac{\partial \theta}{\partial \nu_{a}}-\nu_{i} a_{i 1} p_{1} \theta=0 \quad \text { on } \mathbb{R} \times \partial \omega .
$$

Equation (3.3) can be established via the perturbed test functions method [8], using the test functions of the form $\Phi_{\varepsilon}(x)=\Phi\left(x_{1}\right)-\varepsilon \log \theta\left(x / \varepsilon, x_{1}, p_{1}\right)+o(\varepsilon)$, $p_{1}=\Phi^{\prime}\left(x_{1}\right)$. Constructing the test functions near the bases of the cylinder is more complicated. For simplicity, we assume that the interval $(0, L)$ contains the whole number of micro-periods, $\varepsilon$; i.e., $L / \varepsilon$ is an integer. We concentrate our attention on one base $x_{1}=0$, using the same reasonings for the other base $x_{1}=$ $L$. Considering the ansatz

$$
\Phi_{\varepsilon}=\Phi\left(x_{1}\right)-\varepsilon \log \left(v(x / \varepsilon) \theta\left(x / \varepsilon, x_{1}, p_{1}\right)\right)+o(\varepsilon), \quad p_{1}=\Phi^{\prime}\left(x_{1}\right),
$$

and freezing the slow variable $x_{1}=0$ yields the equation (in the semi-infinite cylinder)

$$
a_{i j} \frac{\partial^{2} v}{\partial y_{i} \partial y_{j}}+\left(b_{j}+2 a_{i j} \frac{\partial \log \theta}{\partial y_{i}}-2 a_{1 j} p_{1}\right) \frac{\partial v}{\partial y_{j}}=0 \quad \text { in }(0,+\infty) \times \omega
$$

with the boundary conditions

$$
\frac{\partial v}{\partial \nu_{a}}=0 \quad \text { on }(0,+\infty) \times \partial \omega
$$

and

$$
\frac{\partial v}{\partial \nu_{a}}+\left(g_{-}\left(y^{\prime}\right)+a_{11} p_{1}+\frac{\partial \log \theta}{\partial \nu_{a}}\right) v=\bar{g}_{-}\left(p_{1}\right) v \quad \text { on }\{0\} \times \omega .
$$

The number $\bar{g}_{-}\left(p_{1}\right)$ and the function $v$ are unknown in problem (3.7)-(3.9), and the solution $\bar{g}_{-}\left(p_{1}\right)$ defines an effective boundary condition at $x_{1}=0$ in problem (3.3). More precisely, we seek a constant $\bar{g}_{-}\left(p_{1}\right)$ such that problem (3.7)-(3.9) has a bounded positive solution converging to a positive constant as $y_{1} \rightarrow+\infty$. This is a kind of the Steklov spectral problem studied in detail in Section 4. Some of its main features are described in the following statement. 
Theorem 3.1. There is a continuous strictly increasing function $\bar{g}_{-}^{*}(h)$ on $\left[\min _{p_{1}} \bar{H}\left(0, p_{1}\right),+\infty\right)$ (which grows not slower than a linear function as $h \rightarrow+\infty$ ) such that problem (3.7)-(3.9) has a bounded positive solution that converges to a positive constant as $y_{1} \rightarrow+\infty$ if and only if one of the following two conditions is fulfilled:

(i) $\frac{\partial \bar{H}}{\partial p_{1}}\left(0, p_{1}\right) \geq 0$ and $\bar{g}_{-}\left(p_{1}\right)=\bar{g}_{-}^{*}\left(\bar{H}\left(0, p_{1}\right)\right)$, or

(ii) $\frac{\partial \bar{H}}{\partial p_{1}}\left(0, p_{1}\right)<0$ and $\bar{g}_{-}\left(p_{1}\right)<\bar{g}_{-}^{*}\left(\bar{H}\left(0, p_{1}\right)\right)$.

The similar result holds for $x_{1}=L$ with some function $\bar{g}_{+}^{*}(h)$.

Now, for a given smooth function $\Phi\left(x_{1}\right)$, we use test functions of the form (3.6) and (as usual in viscosity solutions theory) consider the local maxima and minima points of $W_{\varepsilon}-\Phi_{\varepsilon}$ to conclude (passing to the limit $\varepsilon \rightarrow 0$ ) that

- if $W-\Phi$ attains its local maximum (in $[0, L]$ ) at $x_{1}=0$, then either $\bar{H}\left(0, \Phi^{\prime}(0)\right) \leq \lambda$ or $\bar{g}_{-}\left(\bar{H}\left(0, \Phi^{\prime}(0)\right)\right) \geq 0$, and $-\frac{\partial \bar{H}}{\partial p_{1}}\left(0, \Phi^{\prime}(0)\right) \leq 0 ;$

- if $W-\Phi$ attains its local minimum at $x_{1}=0$, then either $\bar{H}\left(0, \Phi^{\prime}(0)\right) \geq \lambda$ or $\bar{g}_{-}\left(\bar{H}\left(0, \Phi^{\prime}(0)\right)\right) \leq 0$.

Introduce $\bar{h}_{-}$as the unique solution of the equation $g_{-}\left(\bar{h}_{-}\right)=0$ if it exists and set $\bar{h}_{-}=-\infty$ otherwise. Define $\bar{h}_{+}$in a similar way (via the function $\bar{g}_{+}^{*}(h)$ ). Then, using formalism of the viscosity solutions theory, we can write the effective problem for $\lambda$ and $W$ as equation (3.3) with the boundary conditions in the form of inequalities (sub- and supersolution property):

$$
-\bar{H}\left(x_{1}, W^{\prime}\left(x_{1}\right)\right)+\bar{h}_{ \pm} \leq 0 \text { and } \mp \frac{\partial \bar{H}}{\partial p_{1}}\left(x_{1}, W^{\prime}\left(x_{1}\right)\right) \leq 0 \text { at } x_{1}=(L \pm L) / 2,
$$

and

$$
-\bar{H}\left(x_{1}, W^{\prime}\left(x_{1}\right)\right)+\bar{h}_{ \pm} \geq 0 \text { at } x_{1}=(L \pm L) / 2,
$$

both understood in the viscosity solutions sense.

Proposition 3.2. There exists a unique constant $\lambda=\bar{\lambda}$ (additive eigenvalue) such that problem (3.3), (3.10), (3.11) has a continuous viscosity solution $W$. Moreover, $\bar{\lambda}$ is given by the formula

$$
\bar{\lambda}=\max \left\{\bar{h}_{ \pm}, \max _{x_{1} \in[0, L]} \min _{p_{1}} \bar{H}\left(x_{1}, p_{1}\right)\right\} .
$$

Proof. First observe that every viscosity solution of (3.3) satisfies the equation pointwisely almost everywhere, therefore

$$
\lambda \geq \max _{x_{1} \in[0, L]} \min _{p_{1}} \bar{H}\left(p_{1}, x_{1}\right) .
$$

Moreover, $W$ is continuously differentiable everywhere except at the most finite number of points in $(0, L)$ where $W$ is continuous and one-side limits of derivatives 
exist. It follows, in particular, that $\bar{H}\left(x_{1}, W^{\prime}\left(x_{1}\right)\right)=\lambda$ at endpoints $x_{1}=(L \pm$ $L) / 2$. Show that $\lambda \geq \max \left\{\bar{h}_{ \pm}\right\}$. Assume by contradiction that $\lambda<\max \left\{\bar{h}_{ \pm}\right\}$ and consider, for definiteness, the case when $\max \left\{\bar{h}_{ \pm}\right\}=\bar{h}_{-}$. We use the test function $\Phi\left(x_{1}\right):=\left(p_{1}+\delta\right) x_{1}$, where $p_{1}$ is the maximal solution of the equation $\bar{H}\left(0, p_{1}\right)=\lambda\left(=\bar{H}\left(0, W^{\prime}(0)\right)\right)$ and $\delta>0$, to verify the subsolution property at $x_{1}=0$. This yields $\bar{H}\left(0, p_{1}+\delta\right) \leq \lambda$ or $\bar{H}\left(0, p_{1}+\delta\right) \geq \bar{h}_{-}$, but both inequalities are broken for sufficiently small $\delta$. Thus, $\lambda \geq \bar{\lambda}$.

Now assume that $\lambda>\bar{\lambda}$, in particular $\lambda>\bar{h}_{-}$. Set $p_{1}^{-}:=W^{\prime}(0)$. If $\frac{\partial \bar{H}}{\partial p_{1}}\left(0, p_{1}^{-}\right)>0$, then considering the test function $\Phi\left(x_{1}\right):=\left(p_{1}-\delta\right) x_{1}$ with sufficiently small $\delta>0$ we have $\bar{H}\left(0, \Phi^{\prime}(0)\right)<\lambda$, so that by $(3.11) \Phi^{\prime}(0)$ satisfies $\bar{H}\left(0, \Phi^{\prime}(0)\right) \leq \bar{h}_{-}$. Actually, $\lambda-O(\delta) \leq \bar{h}_{-}$is a contradiction. Thus, $\frac{\partial \bar{H}}{\partial p_{1}}\left(0, W^{\prime}(0)\right) \leq 0$. The similar reasoning yields $\frac{\partial \bar{H}}{\partial p_{1}}\left(0, W^{\prime}(L)\right) \geq 0$. Observe also that these inequalities are strict, otherwise $\lambda=\bar{H}\left(x_{1}, W^{\prime}\left(x_{1}\right)\right)=$ $\min _{p_{1}} \bar{H}\left(x_{1}, p_{1}\right) \leq \bar{\lambda}$, where $x_{1}=0$ or $x_{1}=L$. It follows that at some point $\xi \in(0, L)$ we have $\lim _{x_{1} \rightarrow \xi-0} W^{\prime}\left(x_{1}\right)<\lim _{x_{1} \rightarrow \xi+0} W^{\prime}\left(x_{1}\right)$. But then $W\left(x_{1}\right)$ does not satisfy the equation $\bar{H}\left(x_{1}, W^{\prime}\left(x_{1}\right)\right)=\lambda$ at $\xi$ (in viscosity sense).

Next we state the main result describing the asymptotic behavior of the first eigenpair.

Theorem 3.3. Assume that conditions (2.5), (2.6) are satisfied. Then the first eigenvalue $\lambda_{\varepsilon}$ converges as $\varepsilon \rightarrow 0$ to $\bar{\lambda}$ given by (3.12). The scaled $\log$ transformations $W_{\varepsilon}=-\varepsilon \log u_{\varepsilon}$ of the first eigenfunctions $u_{\varepsilon}$ (normalized by $\max u_{\varepsilon}=1$ ) converge uniformly (up to extracting a subsequence) to a viscosity solution $W\left(x_{1}\right)$ of problem (3.3), (3.10), (3.11).

\section{Construction of boundary layers near bases}

Consider the following problem in a semi-infinite cylinder: find a number $\bar{g}$ and positive bounded function $v$ satisfying

$$
\begin{aligned}
a_{i j} \frac{\partial^{2} v}{\partial y_{i} \partial y_{j}}+b_{j} \frac{\partial v}{\partial y_{j}} & =0 & & \text { in }(0,+\infty) \times \omega, \\
\frac{\partial v}{\partial \nu_{a}}+g\left(y^{\prime}\right) v & =\bar{g} v & & \text { on }\{0\} \times \omega, \\
\frac{\partial v}{\partial \nu_{a}} & =0 & & \text { on }(0,+\infty) \times \partial \omega .
\end{aligned}
$$

We assume that the coefficients $a_{i j}(y), b_{j}(y), c(y) \in C^{3}(\mathbb{R} \times \bar{\omega})$ are 1-periodic in $y_{1}$, $a_{i j}(y)$ admit the symmetry $a_{i j}=a_{j i}$ and satisfy the uniform ellipticity condition, $g\left(y^{\prime}\right) \in C^{2}(\bar{\omega})$ is a given function. The qualitative features of problem (4.1)-(4.3) are determined by the sign of the so-called longitudinal drift defined as follows. For the equation

$$
\frac{\partial^{2}}{\partial y_{i} \partial y_{j}}\left(a_{i j} \theta^{*}\right)-\frac{\partial}{\partial y_{j}}\left(b_{j} \theta^{*}\right)=0 \quad \text { in }(-\infty,+\infty) \times \omega
$$


subject to the boundary condition

$$
\frac{\partial \theta^{*}}{\partial \nu_{a}}+\nu_{j}\left(\frac{\partial}{\partial y_{i}} a_{i j}-b_{j}\right) \theta^{*}=0 \quad \text { on }(-\infty,+\infty) \times \partial \omega
$$

find 1-periodic solution with respect to $y_{1}$. Since the adjoint problem to (4.4), (4.5) has only constant solutions, there is the unique solution of (4.4), (4.5) normalized by

$$
\frac{1}{|\omega|} \int_{(0,1) \times \omega} \theta^{*} d y_{1} d y^{\prime}=1
$$

Then we define the effective longitudinal drift $\bar{b}_{1}$ by

$$
\bar{b}_{1}:=\int_{(0,1) \times \omega} b_{1} \theta^{*} d y_{1} d y^{\prime}-\int_{(0,1) \times \omega} \frac{\partial}{\partial y_{j}}\left(a_{1 j} \theta^{*}\right) d y_{1} d y^{\prime} .
$$

Theorem 4.1. There exists $\bar{g}^{*} \in \mathbb{R}$ such that

1. for $\bar{g}=\bar{g}^{*}$, problem (4.1)-(4.3) has a positive bounded solution $v$ that stabilizes exponentially fast to a constant $v_{\infty}$,

2. if $\bar{b}_{1} \leq 0$, then $v_{\infty}>0$,

3. if $\bar{b}_{1}>0$, then $v_{\infty}=0$.

In the case of $\bar{b}_{1} \leq 0$, the bounded positive solution of (4.1)-(4.3) is unique up to a positive multiplicative constant. Such a solution does not exist if $\bar{g} \neq \bar{g}^{*}$.

In the case of $\bar{b}_{1}>0$, for any $\bar{g}<\bar{g}^{*}$ there is a bounded positive solution of (4.1)-(4.3) stabilizing exponentially fast to some $v_{\infty}>0$. There are no bounded positive solutions of (4.1)-(4.3) for $\bar{g}>\bar{g}^{*}$.

Proof. Consider the auxiliary spectral problem: find the first eigenvalue $\Lambda=$ $\Lambda(N)$ (corresponding to a positive eigenfunction) of the Steklov eigenvalue problem

$$
a_{i j} \frac{\partial^{2} v_{N}}{\partial y_{i} \partial y_{j}}+b_{j} \frac{\partial v_{N}}{\partial y_{j}}=0 \quad \text { in }(0, N) \times \omega,
$$

with spectral parameter $\Lambda$ in the boundary condition

$$
\frac{\partial v_{N}}{\partial \nu_{a}}+g\left(y^{\prime}\right) v_{N}=\Lambda v_{N} \quad \text { on }\{0\} \times \omega
$$

Equation (4.8) is also supplied with the Dirichlet condition

$$
v_{N}=0 \quad \text { on }\{N\} \times \omega,
$$

and the Neumann condition

$$
\frac{\partial v_{N}}{\partial \nu_{a}}=0
$$

on the lateral surface $(0, N) \times \partial \omega$. 
By the Krein-Rutman theorem, problem (4.8)-(4.11) has the unique eigenvalue $\Lambda=\Lambda(N)$, the principal eigenvalue corresponding to a positive eigenfunction $v_{N}(y)$. Note that $v_{N}$ attains its maximum at a point on $\{0\} \times \omega$, then condition (4.9) at this point yields $\Lambda(N) \geq \min _{\bar{\omega}} g\left(y^{\prime}\right)$. Also, $\Lambda(N)$ enjoys the monotonicity property: $\Lambda\left(N_{1}\right) \leq \Lambda\left(N_{2}\right)$ if $N_{1}>N_{2}>0$. Indeed, observe that the positive function $\tilde{v}:=v_{N_{2}} / v_{N_{1}}$ satisfies a convection-diffusion equation in $\left(0, N_{2}\right) \times \omega$ with the Neumann boundary condition $\frac{\partial \tilde{v}}{\partial \nu_{a}}=0$ on $\left(0, N_{2}\right) \times \partial \omega$, the Dirichlet condition $\tilde{v}=0$ on $\left\{L_{2}\right\} \times \omega$ and the condition $\frac{\partial \tilde{v}}{\partial \nu_{a}}=\left(\Lambda\left(N_{2}\right)-\Lambda\left(N_{1}\right)\right) \tilde{v}$ on $\{0\} \times \omega$. Since $\tilde{v}$ attains its positive maximum on $\{0\} \times \omega$, we get $\Lambda\left(N_{2}\right)-$ $\Lambda\left(N_{1}\right) \geq 0$. Thus there exists

$$
\bar{g}^{*}=\lim _{N \rightarrow+\infty} \Lambda(N) .
$$

Normalize the functions $v_{N}$ by setting $\min _{y \in\{0\} \times \bar{\omega}} v_{N}(y)=1$ and pass to the limit as $N \rightarrow \infty$ using elliptic estimates to find a bounded positive solution of (4.1)-(4.3) for $\bar{g}=\bar{g}^{*}$.

Consider now a positive solution $v$ of (4.1)-(4.3) in the case when $\bar{b}_{1} \leq 0$. Here, according to [10], equation (4.1) with boundary condition (4.3) and prescribed Dirichlet data on $\{0\} \times \omega$ has a unique bounded solution and it stabilizes exponentially fast to a constant as $y_{1} \rightarrow+\infty$. Thus $v$ stabilizes to a positive constant. If $\tilde{v}$ is another positive solution, then their ratio $v / \tilde{v}$ satisfies a convectiondiffusion equation with the boundary condition $\frac{\partial}{\partial \nu_{a}}(v / \tilde{v})=0$ on $\{0\} \times \omega$ and tends to a constant as $y_{1} \rightarrow+\infty$. Consequently, the function $v / \tilde{v}$ is necessarily constant. The same factorization argument shows that if (4.1)-(4.3) has a bounded positive solution, then $\bar{g}=\bar{g}^{*}$.

In the case of $\bar{b}_{1}>0$, for every prescribed positive Dirichlet data on $\{0\} \times \omega$ there is a solution of (4.8) satisfying (4.3) and vanishing as $y_{1} \rightarrow+\infty$ (see [10]). Such a solution can be chosen to dominate all functions $v_{N}$ and therefore their limit $v$. Thus, for $\bar{g}=\bar{g}^{*}$, there is a positive solution $v^{*}$ such that $v^{*} \rightarrow 0$ as $y_{1} \rightarrow$ $+\infty$. Let us consider $\bar{g}<\bar{g}^{*}$ and construct a solution $\tilde{v}_{N}$ of $(4.8)$ in $(0, N) \times$ $\omega$ satisfying also $(4.3)$ on $(0, N) \times \partial \omega$. We seek $\tilde{v}_{N}$ in the form $\tilde{v}_{N}=w_{N}\left(v^{*}+\right.$ $\delta$ ), where $v^{*}$ is a solution of (4.1)-(4.3) for $\bar{g}=\bar{g}^{*}$ and $\delta$ is a positive constant to be chosen later. Substituting this representation in (4.8) and (4.3) leads to a convection-diffusion equation for $w_{N}$ and the Neumann condition $\frac{\partial w_{N}}{\partial \nu_{a}}=0$ on $(0, N) \times \partial \omega$. We also prescribe the Dirichlet data $w_{N}=1 /\left(v^{*}+\delta\right)$ on $\{N\} \times \omega$. Finally, we want $w_{N}\left(v^{*}+\delta\right)$ to satisfy (4.2) on $\{0\} \times \omega$ that yields

$$
\frac{\partial w_{N}}{\partial \nu_{a}}+\left(\frac{\delta}{v^{*}+\delta}\left(g\left(y^{\prime}\right)-\bar{g}^{*}\right)+\bar{g}^{*}-\bar{g}\right) w_{N}=0 .
$$

For sufficiently small $\delta>0$, the factor in front of $w_{N}$ becomes positive, $\frac{\delta}{v^{*}+\delta}\left(g\left(y^{\prime}\right)-\bar{g}^{*}\right)+\bar{g}^{*}-\bar{g}>0$ on $\{0\} \times \omega$. For this $\delta$ there exists a unique positive solution $w_{N}$ such that $\tilde{v}_{N}$ is now well-defined. Moreover, applying the maximum principle, we get $w_{N} \leq \max _{\{N\} \times \omega} \frac{1}{v^{*}+\delta} \leq \frac{1}{\delta}$ in $(0, N) \times \omega$, i.e., $\tilde{v}_{N} \leq C$ with $C$ independent of $N$. Also, according to [10], there exists a positive function $u_{0}$ satisfying $(4.1)$ in $(0,+\infty) \times \omega$, boundary condition $(4.3)$ on $(0,+\infty) \times \partial \omega$, 
and such that $u_{0}=0$ when $y_{1}=0$ and $u_{0} \rightarrow 1$ as $y_{1} \rightarrow+\infty$. Then, by the maximum principle, $\tilde{v}_{N}>u_{0}$. Thus, taking the limit $N \rightarrow+\infty$ (along some sequence) yields a bounded positive solution $\tilde{v}$ of (4.1)-(4.3) which also remains bounded away from 0 as $y_{1} \rightarrow+\infty$. Moreover, $\tilde{v} \geq c>0$ on $\{0\} \times \omega$. If $\tilde{v}=0$ at a point on $\{0\} \times \omega$, then by Hopf's boundary lemma, $\frac{\partial \tilde{v}}{\partial \nu_{a}}<0$ at this point, which contradicts (4.2).

Finally, assume by contradiction that for the case $\bar{b}_{1}>0$ there exists a bounded positive solution $v$ for $\bar{g}>\bar{g}^{*}$. Then, rewriting the solution $v^{*}$ (that corresponds to $\bar{g}^{*}$ and vanishes as $\left.y_{1} \rightarrow+\infty\right)$ in the form $v^{*}=\tilde{v}(v+\delta)$ with $\delta>0$, we get a convection-diffusion equation for $\tilde{v}$ in the semi-infinite cylinder $(0,+\infty)$ with the Neumann boundary condition on the lateral surface and the following condition on $\{0\} \times \omega$ :

$$
\frac{\partial \tilde{v}}{\partial \nu_{a}}+\left(\frac{\delta}{v+\delta}\left(g\left(y^{\prime}\right)-\bar{g}\right)+\bar{g}-\bar{g}^{*}\right) \tilde{v}=0 \quad \text { on }\{0\} \times \omega .
$$

On the other hand, $\tilde{v}$ attains its (positive) maximum on $\{0\} \times \omega$ (it vanishes as $\left.y_{1} \rightarrow \infty\right)$, which contradicts the above boundary condition as $\delta>0$ is sufficiently small.

Together with (4.1)-(4.3), we consider the following formally adjoint problem:

$$
\begin{aligned}
\frac{\partial^{2}}{\partial y_{i} \partial y_{j}}\left(a_{i j} w\right)-\frac{\partial}{\partial y_{j}}\left(b_{j} w\right) & =0 & & \text { in }(0,+\infty) \times \omega, \\
\frac{\partial w}{\partial \nu_{a}}+\left(b_{1}-\frac{\partial}{\partial y_{i}} a_{i 1}+g\left(y^{\prime}\right)\right) w & =\bar{g} w & & \text { on }\{0\} \times \omega, \\
\frac{\partial w}{\partial \nu_{a}}+\nu_{j}\left(\frac{\partial}{\partial y_{i}} a_{i j}-b_{j}\right) w & =0 & & \text { on }(0,+\infty) \times \partial \omega .
\end{aligned}
$$

Problem (4.13)-(4.15) is reduced to that of the form (4.1)-(4.3) by factorizing with 1-periodic in $y_{1}$ solution of problem (4.4), (4.5) where $\theta^{*}$ is normalized by (4.6). Indeed, represent $w$ as $w=\theta^{*} \tilde{w}$ and observe that $\tilde{w}$ satisfies the equation

$$
a_{i j} \frac{\partial^{2}}{\partial y_{i} \partial y_{j}} \tilde{w}+\beta_{j} \frac{\partial}{\partial y_{j}} \tilde{w}=0 \quad \text { in }(0,+\infty) \times \omega
$$

where $\beta_{j}=\frac{2}{\theta^{*}} \frac{\partial}{\partial y_{i}}\left(a_{i j} \theta^{*}\right)-b_{j}$, with the Neumann condition on the lateral surface and the Fourier condition on the base:

$$
\begin{aligned}
\frac{\partial \tilde{w}}{\partial \nu_{a}} & =0 & & \text { on }(0,+\infty) \times \partial \omega, \\
\frac{\partial \tilde{w}}{\partial \nu_{a}}+\left(b_{1}-\frac{\partial}{\theta^{*} \partial y_{i}}\left(a_{i 1} \theta^{*}\right)+g\left(y^{\prime}\right)\right) \tilde{w} & =\bar{g} \tilde{w} & & \text { on }\{0\} \times \omega .
\end{aligned}
$$

Thus, Theorem 4.1 applies also to problem (4.13)-(4.15). Moreover, the constant $\bar{g}^{*}$ is the same as in problem (4.1)-(4.3) due to the fact that it is obtained via 
the limit transition as $N \rightarrow+\infty$ in spectral problems adjoint to problems (4.8)(4.11). Observe also that the effective longitudinal drift $\bar{\beta}_{1}$ for problem (4.13)(4.15) is $\bar{\beta}_{1}=-\bar{b}_{1}$. To this end, note that $\theta^{*}$ satisfies

$$
\frac{\partial^{2}}{\partial y_{i} \partial y_{j}}\left(a_{i j} \theta^{*}\right)-\frac{\partial}{\partial y_{j}}\left(\beta_{j} \theta^{*}\right)=0 \quad \text { in }(-\infty,+\infty) \times \omega
$$

and

$$
\frac{\partial \theta^{*}}{\partial \nu_{a}}+\nu_{j}\left(\frac{\partial}{\partial y_{i}} a_{i j}-\beta_{j}\right) \theta^{*}=0 \quad \text { on }(-\infty,+\infty) \times \partial \omega .
$$

Consequently,

$$
\bar{\beta}_{1}=\int_{(0,1) \times \omega}\left(2 \frac{\partial}{\partial y_{j}}\left(a_{1 j} \theta^{*}\right)-b_{1} \theta^{*}-\frac{\partial}{\partial y_{j}}\left(a_{1 j} \theta^{*}\right)\right) d y_{1} d y^{\prime}=-\bar{b}_{1} .
$$

These results are summarized in

Theorem 4.2. Let $\bar{b}_{1}$ be given by (4.7). Problem (4.13)-(4.15) has a unique (up to multiplication by a positive constant) bounded positive solution for $\bar{g}=\bar{g}^{*}$. Moreover, for $\bar{b} \geq 0$, the solution is bounded away from zero while for $\bar{b}_{1}<0$ the solution necessarily decays to zero (exponentially fast) as $y_{1} \rightarrow \infty$. In the case $\bar{b}_{1} \geq 0$, bounded nontrivial solutions of $(4.13)-(4.15)$ do not exist for $\bar{g} \neq \bar{g}^{*}$. If $\bar{b}_{1}<0$, then for every $\bar{g}>\bar{g}^{*}$ there is a bounded solution which is bounded away from zero; there are no bounded solutions if $\bar{g}>\bar{g}^{*}$.

Remark 4.3. According to the results of [9], the solutions $v$ appearing in Theorem 4.1 have the following regularity: $v \in C^{1}([0,+\infty) \times \bar{\omega}) \cap C^{2}((0,+\infty) \times$ $\omega)$. Although the second derivatives of $v$ are in general not bounded near $\{0\} \times$ $\partial \omega$ (because the boundary is not smooth), if $v$ is normalized such that $v \leq 1$, its second derivatives satisfy

$$
\left|\frac{\partial^{2} v}{\partial y_{i} \partial y_{j}}\right| \leq \frac{C}{\operatorname{dist}^{\sigma}(y,\{0\} \times \partial \omega)}
$$

for some $0 \leq \sigma<1$. The same holds for the solution $w$ of the adjoint problem $w$ described in Theorem 4.2. Moreover, since the functions $v$ and $w / \theta^{*}$ converge with exponential rate to constants as $y_{1} \rightarrow+\infty$, their first and second derivatives converge to 0 also with exponential rate (this follows by standard elliptic estimates $[6])$.

Next observation is important for the analysis in Section 5 .

Proposition 4.4. Let $v$ and $w$ be the solutions of (4.1)-(4.3) and (4.13)(4.15) with $\bar{g}=\bar{g}_{v}$ and $\bar{g}=\bar{g}_{w}$, respectively. Then the vector field

$$
\left(b_{j}+2 a_{i j} \frac{\partial \log v}{\partial y_{i}}\right) v w-\frac{\partial}{\partial y_{i}}\left(a_{i j} v w\right)
$$

is divergence-free in the semi-infinite cylinder $(0,+\infty) \times \omega$, its normal component on the lateral surface $(0,+\infty) \times \partial \omega$ vanishes while the normal component on the base $\{0\} \times \omega$ equals $\left(\bar{g}_{v}-\bar{g}_{w}\right) v w$. 
Proof. The claim follows by algebraic manipulations with equations and boundary conditions satisfied by the functions $v$ and $w$.

The remainder of this Section is devoted to the proof of Theorem 3.1.

Proof of Theorem 3.1. The application of Theorem 4.1 to problem (3.7)(3.9) yields the effective constant $\bar{g}_{-}^{*}\left(p_{1}\right)$ whose dependence on $p_{1}$ is examined hereafter. Recall that $\bar{g}_{-}^{*}\left(p_{1}\right)$ is obtained by $\bar{g}_{-}^{*}\left(p_{1}\right)=\lim _{N \rightarrow+\infty} \Lambda\left(N, p_{1}\right)$ via the eigenvalues $\Lambda\left(N, p_{1}\right)$ of the Steklov spectral problems in truncated cylinders (cf. problem (4.8)-(4.11)). Let $v\left(y, N, p_{1}\right)$ be an eigenfunction corresponding to $\Lambda\left(N, p_{1}\right)$. Then the function $\left.\phi:=e^{-p_{1} y_{1}} \theta\left(y, p_{1}, 0\right) v\left(y, N, p_{1}\right)\right)$ solves

$$
a_{i j}(0, y) \frac{\partial^{2} \phi}{\partial y_{i} \partial y_{j}}+b_{j}(0, y) \frac{\partial \phi}{\partial y_{j}}+c(0, y) \phi=\bar{H}\left(0, p_{1}\right) \phi
$$

in $(0, N) \times \omega$ and satisfies

$$
\frac{\partial \phi}{\partial \nu_{a}}=\left\{\begin{array}{ll}
0 & \text { on }(0, N) \times \partial \omega \\
\Lambda\left(N, p_{1}\right) \phi-g_{-}\left(y^{\prime}\right) \phi & \text { on }\{0\} \times \omega
\end{array}, \text { and } \phi=0 \text { on }\{N\} \times \omega .\right.
$$

Now consider an arbitrary $\tilde{p}_{1}$ such that $\bar{H}\left(0, \tilde{p}_{1}\right)=\bar{H}\left(0, p_{1}\right)$ and substitute the representation $\left.\phi=e^{-\tilde{p}_{1} y_{1}} \theta\left(y, 0, \tilde{p}_{1}\right) \tilde{v}\left(y, N, \tilde{p}_{1}\right)\right)$ with $\tilde{v}:=e^{\tilde{p}_{1} y_{1}} \phi / \theta\left(y, 0, \tilde{p}_{1}\right)$ in (4.17), (4.18) to conclude that $\Lambda\left(N, \tilde{p}_{1}\right)=\Lambda\left(N, p_{1}\right)$ and $\tilde{v}$ is a solution of the Steklov problem in the truncated cylinder with $\tilde{p}_{1}$ in place of $p_{1}$. Thus, $\Lambda\left(N, p_{1}\right)$ is expressed as a function of $\bar{H}\left(0, p_{1}\right)$. The same property holds for $\bar{g}_{-}^{*}\left(p_{1}\right)$ and we write, with a little abuse of notation,

$$
\bar{g}_{-}^{*}=\bar{g}_{-}^{*}(h), h=\bar{H}\left(0, p_{1}\right) .
$$

It is straightforward to verify that the effective longitudinal drift $\bar{b}_{1}$ for problem (3.7)-(3.9) is given by

$$
\bar{b}_{1}\left(p_{1}\right)=-\frac{\partial \bar{H}}{\partial p_{1}}\left(0, p_{1}\right)
$$

Also, the continuity of $\bar{g}_{-}^{*}(h)$ is established directly by resolving the equation $h=$ $\bar{H}\left(0, p_{1}\right)$ with $p_{1}$ such that $\frac{\partial \bar{H}}{\partial p_{1}}\left(0, p_{1}\right) \geq 0$ and considering problem (3.7)-(3.9).

We proceed with monotonicity of $\bar{g}_{-}^{*}(h)$. Given $h$ and $\tilde{h}>h$ such that there are the solutions $p_{1}$ and $\tilde{p}_{1}>p_{1}$ of the equations $\bar{H}\left(0, p_{1}\right)=h, \bar{H}\left(0, \tilde{p}_{1}\right)=\tilde{h}$. Choose the solution $p_{1}$ such that $\frac{\partial \bar{H}}{\partial p_{1}}\left(0, p_{1}\right) \geq 0$. By Theorem 4.1, there is a bounded positive function $v$ that satisfies equation $(4.8)$ in $(0,+\infty) \times \omega$ along with boundary conditions (4.11) on $(0,+\infty) \times \partial \omega$ and (4.9) on $\{0\} \times \omega$, where $\bar{g}_{-}\left(p_{1}\right)=\bar{g}_{-}^{*}\left(\bar{H}\left(0, p_{1}\right)\right)$. Then $\phi:=e^{-p_{1} y_{1}} \theta\left(y, 0, p_{1}\right) v$ satisfies $(4.17)$ in $(0,+\infty) \times$ $\omega$ along with the boundary conditions $\frac{\partial \phi}{\partial \nu_{a}}=0$ and $\frac{\partial \phi}{\partial \nu_{a}}+g_{-}\left(y^{\prime}\right) \phi=\bar{g}_{-}^{*}(h) \phi$ on the lateral surface and the base, respectively. Replacing $p_{1}$ by $\tilde{p}_{1}$, we get another 
bounded positive function $\tilde{v}$ whose properties are analogous to those of $v$ and define $\tilde{\phi}$ by $\tilde{\phi}=e^{-\tilde{p}_{1} y_{1}} \theta\left(y, 0, \tilde{p}_{1}\right) \tilde{v}$. Then the ratio $\psi=\tilde{\phi} / \phi$ satisfies

$$
a_{i j}(0, y) \frac{\partial^{2} \psi}{\partial y_{i} \partial y_{j}}+\left(b_{j}(0, y)+2 a_{i j}(0, y) \frac{\partial \log \phi}{\partial y_{i}}\right) \frac{\partial \psi}{\partial y_{j}}-(\tilde{h}-h) \psi=0
$$

in $(0,+\infty) \times \omega$ and the boundary conditions $\frac{\partial \psi}{\partial \nu_{a}}=0$ on $(0,+\infty) \times \partial \omega$,

$$
\frac{\partial \psi}{\partial \nu_{a}}=\left(\bar{g}_{-}(\tilde{h})-g_{-}(h)\right) \psi \quad \text { on }\{0\} \times \omega .
$$

Since $\psi$ (exponentially) decays to 0 as $y_{1} \rightarrow+\infty$, its maximum is necessarily attained at a point on $\{0\} \times \omega$. Then the strict inequality $\bar{g}_{-}(\tilde{h})-g_{-}(h)>0$ follows by Hopf's boundary lemma.

To obtain a lower bound for $\bar{g}_{-}^{*}(h)$ as $h \rightarrow+\infty$, choose an arbitrary $p_{1}$ solving the equation $\bar{H}\left(0, p_{1}\right)=h$ and consider the functions $\phi=$ $e^{-p_{1} y_{1}} \theta\left(y, 0, p_{1}\right) v\left(y, N, p_{1}\right)$ (in truncated cylinders). Multiply (4.17) by $\phi$ and integrate over $(0, N) \times \omega$ to obtain via integration by parts

$$
\begin{aligned}
\int_{\{0\} \times \omega} \frac{\partial \phi}{\partial \nu_{a}} \phi d y^{\prime}= & \int_{(0, N) \times \omega} a_{i j}(0, y) \frac{\partial \phi}{\partial y_{i}} \frac{\partial \phi}{\partial y_{j}} d y \\
& +\int_{(0, N) \times \omega}\left(\frac{\partial a_{i j}(0, y)}{\partial y_{i}}-b_{j}(0, y)\right) \frac{\partial \phi}{\partial y_{j}} \phi d y \\
& +\int_{(0, N) \times \omega}(h-c(0, y)) \phi^{2} d y .
\end{aligned}
$$

Using (4.21) in (4.22) and applying the Cauchy-Schwarz inequality, we get

$$
\left(\Lambda\left(N, p_{1}\right)+C_{-}\right) \int_{\{0\} \times \omega} \phi^{2} d y^{\prime} \geq \int_{(0, N) \times \omega}\left(\frac{\gamma}{2}|\nabla \phi|^{2}+\left(h-C_{1}\right) \phi^{2}\right) d y,
$$

where $C_{-}=\max _{\omega}\left|g_{-}\right|, \gamma$ is the ellipticity constant of $a_{i j}$ and $C_{1}$ depends on $\gamma$ and $L^{\infty}$-bounds for $\left|\frac{\partial a_{i j}(0, y)}{\partial y_{i}}\right|,\left|b_{j}(0, y)\right|$ and $|c(0, y)|$. Thus,

$$
\Lambda\left(N, p_{1}\right)+C_{-} \geq \inf \frac{1}{\varphi^{2}(0)} \int_{0}^{N}\left(\frac{\gamma}{2}\left|\varphi^{\prime}(t)\right|^{2}+\left(h-C_{1}\right) \varphi^{2}(t)\right) d t
$$

where the infimum is taken over all $\varphi \in H^{1}(0, N)$ such that $\varphi(N)=0$. Solving the latter minimization problem, we derive the bound $\Lambda\left(N, p_{1}\right) \geq \sqrt{\gamma\left(h-C_{1}\right) / 2}-$ $C_{-}$, the required lower bound for $\bar{g}_{-}^{*}(h)$ follows by passing to the limit $N \rightarrow+\infty$. Theorem 3.1 is proved.

\section{Two-term asymptotic formulas for the first and higher order eigenvalues}

In this section, we consider the asymptotic behavior of the solutions of spectral problem (2.4) under a structure assumption on the effective problem (3.3), (3.10), (3.11). We assume here that

the maximum in (3.12) is strict, it is attained at an inner point $\xi \in(0, L)$, 


$$
\text { and }-V:=\left(\min _{p_{1}} \bar{H}\left(x_{1}, p_{1}\right)\right)^{\prime \prime}<0 \text { at } x_{1}=\xi .
$$

Under this condition we establish the first two terms in the asymptotic expansion of the eigenvalues. Note that the technique developed here allows us to treat the first eigenvalue as well as higher order ones while the method in Section 3 is based on the maximum principle and therefore can be applied only to the first eigenpair.

Under condition (5.1) there is a twice continuously differentiable function $p_{1}\left(x_{1}\right)$ such that

$$
\begin{aligned}
& \bar{H}\left(x_{1}, p_{1}\left(x_{1}\right)\right)<\bar{H}\left(\xi, p_{1}(\xi)\right)(=\bar{\lambda}) \quad \text { in }[0, L] \backslash\{\xi\} \\
& \bar{H}\left(x_{1}^{ \pm}, p_{1}\left(x_{1}^{ \pm}\right)\right)>h_{ \pm}, \mp \frac{\partial \bar{H}}{\partial p_{1}}\left(x_{1}^{ \pm}, p_{1}\left(x_{1}^{ \pm}\right)\right)>0 \text { at the ends } x_{1}^{ \pm}=\frac{L \pm L}{2} \\
& \left(\bar{H}\left(x_{1}, p_{1}\left(x_{1}\right)\right)\right)^{\prime \prime}=-V<0 \text { at } x_{1}=\xi .
\end{aligned}
$$

Note that the first inequality in (5.2) implies that

$$
\bar{g}_{-}^{*}\left(p_{1}(0)\right)>0, \quad \bar{g}_{+}^{*}\left(p_{1}(L)\right)>0 .
$$

Let $Q\left(x_{1}\right)$ be a primitive function of $p_{1}\left(x_{1}\right), Q^{\prime}\left(x_{1}\right)=p_{1}\left(x_{1}\right)$. Represent the eigenfunctions $u_{\varepsilon}$ with their corresponding eigenvalues $\lambda_{\varepsilon}$ in the form

$$
u_{\varepsilon}=e^{-Q\left(x_{1}\right) / \varepsilon} \theta\left(x / \varepsilon, x_{1}, p_{1}\left(x_{1}\right)\right) \phi_{\varepsilon}, \quad \lambda_{\varepsilon}=\bar{\lambda}-\varepsilon \mu_{\varepsilon},
$$

where $\theta\left(y, x_{1}, p_{1}\right)$ is the 1-periodic in $y_{1}$ positive solution of (3.4), (3.5) normalized by $\int_{(0,1) \times \omega} \theta\left(y, x_{1}, p_{1}\right) d y=1$. Then the equation $\mathcal{L}_{\varepsilon} u_{\varepsilon}=\lambda_{\varepsilon} u_{\varepsilon}$ rewrites as

$$
\mathcal{L}_{\varepsilon}^{(1)} \phi_{\varepsilon}-\frac{1}{\varepsilon} U\left(x_{1}\right) \phi_{\varepsilon}=-\mu_{\varepsilon} \phi_{\varepsilon}+\mathcal{R}_{\varepsilon}^{(1)} \phi_{\varepsilon}
$$

where

$$
\begin{aligned}
U\left(x_{1}\right) & =\bar{\lambda}-\bar{H}\left(p_{1}\left(x_{1}\right), x_{1}\right), \\
\mathcal{L}_{\varepsilon}^{(1)} \phi_{\varepsilon} & =\varepsilon a_{i j}\left(x_{1}, x / \varepsilon\right) \frac{\partial^{2} \phi_{\varepsilon}}{\partial x_{i} \partial x_{j}}+\tilde{b}_{j}\left(x_{1}, x / \varepsilon\right) \frac{\partial \phi_{\varepsilon}}{\partial x_{j}}, \\
\tilde{b}_{j}\left(x_{1}, y\right) & =b_{j}\left(x_{1}, y\right)+2 a_{i j}\left(x_{1}, y\right) \frac{\partial \log \theta\left(y, x_{1}, p_{1}\left(x_{1}\right)\right)}{\partial y_{i}}-2 a_{1 j}\left(x_{1}, y\right) p_{1}\left(x_{1}\right), \\
\mathcal{R}_{\varepsilon}^{(1)} \phi_{\varepsilon} & =\zeta_{\varepsilon}^{(1)} \phi_{\varepsilon}+\varepsilon \eta_{j, \varepsilon}^{(1)} \frac{\partial \phi_{\varepsilon}}{\partial x_{j}},
\end{aligned}
$$

and $\zeta_{\varepsilon}^{(1)}, \eta_{j, \varepsilon}^{(1)}$ are uniformly bounded functions. Multiply (5.7) by the function $\theta^{*}\left(x / \varepsilon, x_{1}, p_{1}\left(x_{1}\right)\right)$, where $\theta^{*}\left(y, x_{1}, p_{1}\right)$ is the positive 1 -periodic in $y_{1}$ solution of the adjoint to (3.7) equation

$$
\frac{\partial^{2}}{\partial y_{i} \partial y_{j}}\left(a_{i j} \theta^{*}\right)-\frac{\partial}{\partial y_{j}}\left(\tilde{b}_{j} \theta^{*}\right)=0 \quad \text { in } \mathbb{R} \times \omega
$$


with the boundary condition

$$
\frac{\partial \theta^{*}}{\partial \nu_{a}}+\nu_{j}\left(\frac{\partial}{\partial y_{i}} a_{i j}-\tilde{b}_{j}\right) \theta^{*}=0 \quad \text { on } \mathbb{R} \times \partial \omega
$$

normalized by

$$
\frac{1}{|\omega|} \int_{(0,1) \times \omega} \theta^{*}\left(y, x_{1}, p_{1}\left(x_{1}\right)\right) d y=1
$$

After rearranging, we obtain

$$
\varepsilon \frac{\partial}{\partial x_{i}}\left(\theta^{*} a_{i j} \frac{\partial \phi_{\varepsilon}}{\partial x_{j}}\right)+B \cdot \nabla \phi_{\varepsilon}-\frac{1}{\varepsilon} U\left(x_{1}\right) \theta^{*} \phi_{\varepsilon}=-\mu_{\varepsilon} \theta^{*} \phi_{\varepsilon}+\mathcal{R}_{\varepsilon}^{(2)} \phi_{\varepsilon},
$$

where $\mathcal{R}_{\varepsilon}^{(2)} \phi_{\varepsilon}=\zeta_{\varepsilon}^{(2)} \phi_{\varepsilon}-\varepsilon \eta_{j, \varepsilon}^{(2)} \frac{\partial \phi_{\varepsilon}}{\partial x_{j}}$ and $\zeta_{\varepsilon}^{(2)}, \tilde{\eta}_{j, \varepsilon}^{(2)}$ are uniformly bounded functions, $B=B\left(x_{1}, y\right)$ is the vector field with the components

$$
B_{j}\left(x_{1}, y\right)=\theta^{*}\left(x_{1}, y\right) \tilde{b}_{j}\left(x_{1}, y\right)-\frac{\partial}{\partial y_{i}}\left(a_{i j}\left(x_{1}, y\right) \theta^{*}\left(x_{1}, y\right)\right) .
$$

Observe that due to (5.8), (5.9), $\operatorname{div}_{y} B=0$ and the normal component of the vector field $B$ vanishes when $y \in \mathbb{R} \times \partial \omega$.

Representation (5.6) is modified in a small neighborhood of the base $\{0\} \times$ $\varepsilon \omega$ by using another factorization which simplifies the boundary condition for $\phi_{\varepsilon}$ on $\{0\} \times \varepsilon \omega$. Namely, let $v_{-}(y)$ be the solution of (3.7)-(3.9) with $p_{1}=p_{1}(0)$, $\bar{g}_{-}\left(p_{1}\right)=\bar{g}_{-}^{*}\left(p_{1}(0)\right)$ (and $\left.\theta=\theta\left(y, 0, p_{1}(0)\right)\right)$ that converges to 1 (exponentially fast) as $y_{1} \rightarrow+\infty$. Set $\psi_{\varepsilon}=\phi_{\varepsilon} / v_{-}(x / \varepsilon)$, and observe that $v_{-}$satisfies

$$
\left|\mathcal{L}_{\varepsilon}^{(1)} v_{-}(x / \varepsilon)\right| \leq C\left(x_{1} / \varepsilon\right)^{1-\sigma} e^{-c x_{1} / \varepsilon} \leq C_{2},
$$

see Remark 4.3. Then equation (5.7) in terms of new unknown function $\psi_{\varepsilon}$ can be written as

$$
\mathcal{L}_{\varepsilon}^{(1)} \psi_{\varepsilon}+2 a_{i j}\left(x_{1}, x / \varepsilon\right) \frac{\partial \log v_{-}}{\partial y_{i}} \frac{\partial \psi_{\varepsilon}}{\partial x_{j}}-\frac{1}{\varepsilon} U\left(x_{1}\right) \psi_{\varepsilon}=-\mu_{\varepsilon} \psi_{\varepsilon}+\mathcal{R}_{\varepsilon}^{(3)} \psi_{\varepsilon}
$$

(the structure and the properties of $\mathcal{R}_{\varepsilon}^{(3)}$ are analogous to those of $\mathcal{R}_{\varepsilon}^{(k)}, k=1,2$ ), and the boundary condition at the base reads

$$
\frac{\partial \psi_{\varepsilon}}{\partial \nu_{a}}+\frac{\bar{g}_{-}^{*}\left(p_{1}(0)\right)}{\varepsilon} \psi_{\varepsilon}=\tilde{g}_{-}\left(x^{\prime} / \varepsilon\right) \psi_{\varepsilon} \quad \text { on }\{0\} \times \varepsilon \omega,
$$

with some bounded function $\tilde{g}_{-}$. Finally, equation (5.13) is symmetrized similarly to (5.10). To this end, introduce the positive solution $w_{-}(y)$ of the problem adjoint to (3.7)-(3.9) with $p_{1}=p_{1}(0), \bar{g}_{-}\left(p_{1}\right)=0\left(<\bar{g}_{-}^{*}\left(p_{1}(0)\right)\right)$, which satisfies $w_{-}(y) / \theta^{*}\left(y, 0, p_{1}(0)\right) \rightarrow 1$ (exponentially fast) as $y_{1} \rightarrow+\infty$; the existence of such a solution is proved in Section 4. Multiply (5.13) by

$$
\tilde{\theta}^{*}\left(x_{1}, x / \varepsilon\right)=\frac{v_{-}(x / \varepsilon) w_{-}(x / \varepsilon)}{\theta^{*}\left(x / \varepsilon, 0, p_{1}(0)\right)} \theta^{*}\left(x / \varepsilon, x_{1}, p_{1}\left(x_{1}\right)\right)
$$


and rearrange the terms as in (5.10) to find

$$
\varepsilon \frac{\partial}{\partial x_{i}}\left(\tilde{\theta}^{*} a_{i j} \frac{\partial \psi_{\varepsilon}}{\partial x_{j}}\right)+\tilde{B} \cdot \nabla \psi_{\varepsilon}-\frac{1}{\varepsilon} U\left(x_{1}\right) \tilde{\theta}^{*} \psi_{\varepsilon}=-\mu_{\varepsilon} \tilde{\theta}^{*} \psi_{\varepsilon}+\mathcal{R}_{\varepsilon} \psi_{\varepsilon}
$$

where $\tilde{B}=\tilde{B}\left(x_{1}, y\right)$ is the vector field with the components

$$
\tilde{B}_{j}=\tilde{\theta}^{*}\left(x_{1}, y\right)\left(\tilde{b}_{j}\left(x_{1}, y\right)+2 a_{i j}\left(x_{1}, y\right) \frac{\partial \log v_{-}(y)}{\partial y_{i}}\right)-\frac{\partial}{\partial y_{i}}\left(a_{i j}\left(x_{1}, y\right) \tilde{\theta}^{*}\left(x_{1}, y\right)\right),
$$

or $\tilde{B}_{j}=\tilde{B}_{j}^{(1)}+B, \tilde{B}^{(1)}=\tilde{B}^{(1)}\left(x_{1}, y\right)$ is the vector field with the components

$$
\begin{aligned}
\tilde{B}_{j}^{(1)}= & \left(\frac{v_{-}(y) w_{-}(y)}{\theta^{*}\left(y, 0, p_{1}(0)\right)}-1\right) \theta^{*}\left(y, x_{1}, p_{1}\left(x_{1}\right)\right) \tilde{b}_{j}\left(x_{1}, y\right) \\
& +2 a_{i j}\left(x_{1}, y\right) v_{-}(y) w_{-}(y) \frac{\partial \log v_{-}(y)}{\partial y_{i}} \frac{\theta^{*}\left(y, x_{1}, p_{1}\left(x_{1}\right)\right)}{\theta^{*}\left(y, 0, p_{1}(0)\right)} \\
& -\frac{\partial}{\partial y_{i}}\left(a_{i j}\left(x_{1}, y\right)\left(\frac{v_{-}(y) w_{-}(y)}{\theta^{*}\left(y, 0, p_{1}(0)\right)}-1\right) \theta^{*}\left(y, x_{1}, p_{1}\left(x_{1}\right)\right)\right) .
\end{aligned}
$$

Recall that $\operatorname{div}_{y} B=0$, while by Proposition 4.4, we have $\operatorname{div}_{y} \tilde{B}^{(1)}=0$ at $x_{1}=0\left(\tilde{B}_{j}^{(1)}(0, y)=-B_{j}(0, y)+v_{-}(y) w_{-}(y)\left(\tilde{b}_{j}(0, y)+2 a_{i j}(0, y) \frac{\partial \log v_{-}(y)}{\partial y_{i}}\right)-\right.$ $\left.\frac{\partial}{\partial y_{i}}\left(a_{i j}(0, y) v_{-}(y) w_{-}(y)\right)\right)$, hence $\operatorname{div}_{y} \tilde{B}\left(x_{1}, y\right)=\int_{0}^{x_{1}} \frac{\partial}{\partial x_{1}} \operatorname{div}_{y} \tilde{B}^{(1)}(s, y) d s$. At the same time, $\left|\frac{\partial}{\partial x_{1}} \operatorname{div}_{y} \tilde{B}^{(1)}\left(x_{1}, y\right)\right| \leq C e^{-c y_{1}} / y_{1}^{\sigma}$ with $c>0$ and $0 \leq \sigma<1$, see Remark 4.3. Thus,

$$
\begin{aligned}
\left|\operatorname{div} \tilde{B}\left(x_{1}, x / \varepsilon\right)\right| & \leq C_{1}+\frac{1}{\varepsilon}\left|\operatorname{div}_{y} \tilde{B}^{(1)}\left(x_{1}, x / \varepsilon\right)\right| \\
& \leq C_{1}+C \int_{0}^{\infty} \varepsilon^{\sigma} e^{-c x_{1} / \varepsilon} \frac{d x_{1}}{\varepsilon x_{1}^{\sigma}} \leq C_{2} .
\end{aligned}
$$

The similar reasonings lead to the following estimate for the normal component of $\tilde{B}$ :

$$
\left|\tilde{B}_{j}\left(x_{1}, x / \varepsilon\right) \nu_{j}\right| \leq \varepsilon C \quad \text { when } x^{\prime} \in \varepsilon \partial \omega .
$$

Finally, by Proposition 4.4,

$$
-\tilde{B}_{1}\left(x_{1}, x / \varepsilon\right)=\bar{g}_{-}^{*}\left(p_{1}(0)\right) \tilde{\theta}^{*}\left(x_{1}, x / \varepsilon\right) \quad \text { when } x_{1}=0 .
$$

Since the functions $v_{-}(y)$ and $w_{-}(y) / \theta^{*}\left(y, 0, p_{1}(0)\right)$ converge to 1 exponentially fast as $y_{1} \rightarrow \infty$, the above constructed functions $\tilde{\theta}^{*}\left(x_{1}, y\right)$ and $\tilde{\theta}\left(x_{1}, y\right):=$ $v_{-}(y) \theta\left(y, 0, p_{1}(0)\right)$ stabilize, with exponential rate, to the periodic functions $\theta^{*}\left(y, x_{1}, p_{1}\left(x_{1}\right)\right)$ and $\theta\left(y, x_{1}, p_{1}\left(x_{1}\right)\right)$ as $y_{1} \rightarrow+\infty$.

Repeating the above construction near the base $\{L\} \times \varepsilon \omega$, we end up with appropriately modified factors $\tilde{\theta}^{*}, \tilde{\theta}$ and the function $\psi_{\varepsilon}=e^{Q\left(x_{1}\right) / \varepsilon} u_{\varepsilon}(x) / \tilde{\theta}\left(x_{1}, x / \varepsilon\right)$ which satisfies the equation (5.16) in the whole cylinder $(0, L) \times \varepsilon \omega$, where $\mathcal{R}_{\varepsilon} \psi_{\varepsilon}=$ $\zeta_{\varepsilon} \psi_{\varepsilon}+\varepsilon \eta_{j, \varepsilon} \frac{\partial \psi_{\varepsilon}}{\partial x_{j}}$ and $\zeta_{\varepsilon}, \eta_{j, \varepsilon}$ are uniformly bounded functions. Also, $\psi_{\varepsilon}$ satisfies

$$
\frac{\partial \psi_{\varepsilon}}{\partial \nu_{a}}=\tau_{\varepsilon} v_{\varepsilon}
$$


on $(0, L) \times \varepsilon \partial \omega$, with $\left|\tau_{\varepsilon}\right| \leq C$, boundary condition (5.14) on $\{0\} \times \varepsilon \omega$ and an analogous one on $\{L\} \times \varepsilon \omega$. The vector field $\tilde{B}\left(x_{1}, x / \varepsilon\right)$ has uniformly bounded divergence and its normal component satisfies (5.18) on the lateral surface, (5.19) on $\{0\} \times \varepsilon \omega$ and $\tilde{B}_{1}\left(x_{1}, x / \varepsilon\right)=\bar{g}_{+}^{*}\left(p_{1}(0)\right) \tilde{\theta}^{*}\left(x_{1}, x / \varepsilon\right)$ when $x_{1}=L$.

Now introduce the change of variables $z_{1}=\left(x_{1}-\xi\right) / \sqrt{\varepsilon}, z^{\prime}=x^{\prime} / \sqrt{\varepsilon}$ and consider in the rescaled cylinder $\Omega_{\sqrt{\varepsilon}}=(-\xi / \sqrt{\varepsilon},(L-\xi) / \sqrt{\varepsilon}) \times \sqrt{\varepsilon} \omega$ the equation

$$
\begin{aligned}
\tilde{\mathcal{L}}_{\varepsilon} \psi:=-\frac{\partial}{\partial z_{i}}\left(\tilde{\theta}^{*} a_{i j} \frac{\partial \psi}{\partial z_{j}}\right)-\frac{1}{\sqrt{\varepsilon}} \tilde{B} \cdot \nabla \psi & +\frac{1}{\varepsilon} U\left(\xi+\sqrt{\varepsilon} z_{1}\right) \tilde{\theta}^{*} \psi \\
& +\left(\Lambda \tilde{\theta}^{*}+\tilde{\mathcal{R}}_{\varepsilon}\right) \psi=\tilde{\theta}^{*} f,
\end{aligned}
$$

where $\tilde{\theta}^{*}=\tilde{\theta}^{*}\left(x_{1}, x / \varepsilon\right), a_{i j}=a_{i j}\left(x_{1}, x / \varepsilon\right)$ and $\tilde{B}=\tilde{B}\left(x_{1}, x / \varepsilon\right)$ with $x_{1}=\xi+$ $\sqrt{\varepsilon} z_{1}, x^{\prime}=\sqrt{\varepsilon} z^{\prime}, \tilde{\mathcal{R}}_{\varepsilon} \psi=\zeta_{\varepsilon} \psi+\sqrt{\varepsilon} \eta_{j, \varepsilon} \frac{\partial \psi}{\partial z_{j}}$, and $\Lambda>0$ will be chosen later. Supply equation (5.21) with the rescaled (according to the above change of variables) boundary condition (5.20) on the lateral surface, (5.14) on the base $\{-\xi / \sqrt{\varepsilon}\} \times$ $\sqrt{\varepsilon} \omega$ and its counterpart on the second base:

$$
\begin{aligned}
\frac{\partial \psi}{\partial \nu_{a}} & =\sqrt{\varepsilon} \tau_{\varepsilon} \psi & & \text { on }(-\xi / \sqrt{\varepsilon},(L-\xi) / \sqrt{\varepsilon}) \times \sqrt{\varepsilon} \partial \omega, \\
\frac{\partial \psi}{\partial \nu_{a}}+\frac{\bar{g}_{ \pm}^{*}\left(p_{1}(0)\right)}{\sqrt{\varepsilon}} \psi & =\sqrt{\varepsilon} \tilde{g}_{ \pm}\left(x^{\prime} / \varepsilon\right) \psi & & \text { on }\{((L \pm L) / 2-\xi) / \sqrt{\varepsilon}\} \times \sqrt{\varepsilon} \omega,
\end{aligned}
$$

so that $\psi_{\varepsilon}=e^{Q\left(x_{1}\right) / \varepsilon} u_{\varepsilon}(x) /\left.\tilde{\theta}\left(x / \varepsilon, x_{1}\right)\right|_{x_{1}=\xi+\sqrt{\varepsilon} z_{1}, x^{\prime}=\sqrt{\varepsilon} z^{\prime}}$ satisfies (5.22), (5.23) and (5.21) with $f=\left(\Lambda+\mu_{\varepsilon}\right) \psi_{\varepsilon}$.

Multiply (5.21) by $\psi$ and integrate over $\Omega_{\sqrt{\varepsilon}}$ to obtain via integrating by parts

$$
\begin{aligned}
\frac{1}{2 \sqrt{\varepsilon}} \int_{\Omega_{\sqrt{\varepsilon}} \psi^{2} \operatorname{div}_{z} \tilde{B} d z} & -\int_{\partial \Omega_{\sqrt{\varepsilon}}}\left(\tilde{\theta}^{*} \frac{\partial \psi}{\partial \nu_{a}} \psi+\frac{\psi^{2}}{2 \sqrt{\varepsilon}} \tilde{B} \cdot \nu\right) d S+\int_{\Omega_{\sqrt{\varepsilon}}} \tilde{\theta}^{*} a_{i j} \frac{\partial \psi}{\partial z_{i}} \frac{\partial \psi}{\partial z_{j}} d z \\
& +\frac{1}{\varepsilon} \int_{\Omega_{\sqrt{\varepsilon}}}\left(U\left(\xi+\sqrt{\varepsilon} z_{1}\right) \tilde{\theta}^{*}+\varepsilon \Lambda \tilde{\theta}^{*}+\varepsilon \zeta_{\varepsilon}\right) \psi^{2} d z \\
& =\int_{\Omega_{\sqrt{\varepsilon}}}\left(\tilde{\theta}^{*} f-\sqrt{\varepsilon} \eta_{j, \varepsilon} \frac{\partial \psi}{\partial z_{j}}\right) \psi d z
\end{aligned}
$$

By virtue of (5.2)-(5.4), $U\left(\xi+\sqrt{\varepsilon} z_{1}\right) \geq c \varepsilon z_{1}^{2}$ with some $c>0$. Then from (5.24), using (5.22), (5.23), (5.17) (which yields $\left|\operatorname{div}_{z} \tilde{B}\right| \leq C \sqrt{\varepsilon}$ ), (5.18), (5.19) (and its counterpart on the base $\{(L-\xi) / \sqrt{\varepsilon}\} \times \sqrt{\varepsilon} \omega)$, we obtain that

$$
\tilde{\gamma} \int_{\Omega_{\sqrt{\varepsilon}}}\left(\left|\nabla_{z} \psi\right|^{2}+(\Lambda-C) \psi^{2}+z_{1}^{2} \psi^{2}\right) d z \leq \int_{\Omega_{\sqrt{\varepsilon}}} f^{2} d z+\sqrt{\varepsilon} \int_{\partial \Omega_{\sqrt{\varepsilon}}} \psi^{2} d S,
$$

where $\tilde{\gamma}, C$ are independent of $\varepsilon$. Consequently there is some $\Lambda>0$ independent of $\varepsilon$ such that a unique solution $\psi$ of (5.21) (with boundary conditions (5.22), (5.23)) exists for any $f \in L^{2}\left(\Omega_{\sqrt{\varepsilon}}\right)$ and satisfies

$$
\int_{\Omega_{\sqrt{\varepsilon}}}\left(|\nabla \psi|^{2}+\left(1+z_{1}^{2}\right) \psi^{2}\right) d z \leq C \int_{\Omega_{\sqrt{\varepsilon}}} f^{2} d z .
$$


Arguing in a similar way, we obtain the following estimate for the eigenfunction $\psi_{\varepsilon}$ of the operator $\frac{1}{\tilde{\theta}^{*}} \tilde{\mathcal{L}}_{\varepsilon}$ corresponding to an (in general complex) eigenvalue $\Lambda+$ $\mu_{\varepsilon}$ :

$$
\int_{\Omega_{\sqrt{\varepsilon}}}\left(\left|\nabla \psi_{\varepsilon}\right|^{2}+\left(1+z_{1}^{2}\right)\left|\psi_{\varepsilon}\right|^{2}\right) d z \leq C\left(\Lambda+\operatorname{Re}\left(\mu_{\varepsilon}\right)\right) \int_{\Omega_{\sqrt{\varepsilon}}}\left|\psi_{\varepsilon}\right|^{2} d z .
$$

Lemma 5.1. Assume that $\operatorname{Re}\left(\mu_{\varepsilon}\right) \leq C$. Then

(i) $\left|\mu_{\varepsilon}\right|$ is uniformly bounded, and

(ii) every partial limit of $\mu_{\varepsilon}$ as $\varepsilon \rightarrow 0$ is an eigenvalue $\hat{\mu}$ of the one-dimensional problem

$$
-q \hat{\psi}^{\prime \prime}\left(z_{1}\right)+\frac{1}{2} V z_{1}^{2} \hat{\psi}\left(z_{1}\right)+m \hat{\psi}\left(z_{1}\right)=\hat{\mu} \hat{\psi}\left(z_{1}\right), \quad z_{1} \in \mathbb{R},
$$

where $V=-\frac{\partial^{2} \bar{H}}{\partial x_{1}^{2}}\left(p_{1}(\xi), \xi\right)$, the constants $q>0$ and $m$ are given by formulas (5.36) and (5.38) below.

Moreover, under the normalization

$$
\frac{1}{|\sqrt{\varepsilon} \omega|} \int_{\Omega_{\sqrt{\varepsilon}}}\left|\psi_{\varepsilon}\right|^{2} d z=1
$$

the eigenfunctions $\psi_{\varepsilon}$ converge as $\varepsilon \rightarrow 0$, up to a subsequence, to an eigenfunction $\hat{\psi}$ in the following sense:

$$
\frac{1}{|\sqrt{\varepsilon} \omega|} \int_{\Omega_{\sqrt{\varepsilon}}}\left|\psi_{\varepsilon}-\hat{\psi}\right|^{2} d z \rightarrow 0
$$

Proof. It follows from (5.27) and (5.29) that, up to extracting a subsequence, the functions $\psi_{\varepsilon}$ converge to a limit $\hat{\psi}=\hat{\psi}\left(z_{1}\right)$ (where the convergence is understood in the sense (5.30)), moreover

$$
\int_{-\infty}^{\infty}\left(\left|\hat{\psi}^{\prime}\right|^{2}+\left(1+z_{1}^{2}\right)|\hat{\psi}|^{2}\right) d z_{1}<\infty \quad \text { and } \int_{-\infty}^{\infty}|\hat{\psi}|^{2} d z_{1}=1
$$

To identify $\hat{\psi}$, multiply the equation $\tilde{\mathcal{L}}_{\varepsilon} \psi_{\varepsilon}=\left(\Lambda+\mu_{\varepsilon}\right) \tilde{\theta}^{*} \psi_{\varepsilon}$ by an oscillating test function $\rho_{\varepsilon}$ (which will be chosen later) and integrate over $\Omega_{\sqrt{\varepsilon}}$, using integration by parts and rearranging terms, to get

$$
\begin{aligned}
\int_{\Omega_{\sqrt{\varepsilon}}}\left(\left\{\theta^{*} a_{i j}\right\}_{\xi}\right. & \left.\frac{\partial \psi_{\varepsilon}}{\partial z_{i}} \frac{\partial \rho_{\varepsilon}}{\partial z_{j}}+\frac{1}{\sqrt{\varepsilon}} \psi_{\varepsilon}\{B\}_{\xi} \cdot \nabla \rho_{\varepsilon}\right) d z+\int_{\Omega_{\sqrt{\varepsilon}}}\left(\frac{V}{2} z_{1}^{2}+\zeta_{\varepsilon}\right) \tilde{\theta}^{*} \psi_{\varepsilon} \rho_{\varepsilon} d z \\
& +\frac{1}{\sqrt{\varepsilon}} \int_{\Omega_{\sqrt{\varepsilon}}} \psi_{\varepsilon}\left(\left(\tilde{B}-\{B\}_{\xi}\right) \cdot \nabla \rho_{\varepsilon}+\rho_{\varepsilon} \operatorname{div}_{z} \tilde{B}\right) d z \\
& =\mu_{\varepsilon} \int_{\Omega_{\sqrt{\varepsilon}}} \tilde{\theta}^{*} \psi_{\varepsilon} \rho_{\varepsilon} d z+\int_{\partial \Omega_{\sqrt{\varepsilon}}}\left(\tilde{\theta}^{*} \frac{\partial \psi_{\varepsilon}}{\partial \nu_{a}} \rho_{\varepsilon}+\frac{\tilde{B} \cdot \nu}{\sqrt{\varepsilon}} \psi_{\varepsilon} \rho_{\varepsilon}\right) d S
\end{aligned}
$$




$$
\begin{aligned}
& +\int_{\Omega_{\sqrt{\varepsilon}}}\left(\left\{\theta^{*} a_{i j}\right\}_{\xi}-\tilde{\theta}^{*} a_{i j}\right) \frac{\partial \psi_{\varepsilon}}{\partial z_{i}} \frac{\partial \rho_{\varepsilon}}{\partial z_{j}} d z \\
& +\int_{\Omega_{\sqrt{\varepsilon}}}\left(\frac{\varepsilon V z_{1}^{2} / 2-U\left(\xi+\sqrt{\varepsilon} z_{1}\right)}{\varepsilon} \tilde{\theta}^{*} \psi_{\varepsilon}-\sqrt{\varepsilon} \eta_{j, \varepsilon} \frac{\partial \psi_{\varepsilon}}{\partial z_{j}}\right) \rho_{\varepsilon} d z
\end{aligned}
$$

where $\left\{\theta^{*} a_{i j}\right\}_{\xi}=\theta^{*}\left(x_{1}, y\right) a_{i j}\left(x_{1}, y\right)$ and $\{B\}_{\xi}=B\left(x_{1}, y\right)$ with $x_{1}=\xi, y_{1}=\xi / \varepsilon+$ $z_{1} / \sqrt{\varepsilon}, y^{\prime}=z^{\prime} / \sqrt{\varepsilon}$. Let $\rho\left(z_{1}\right)$ be a smooth function with a compact support, say $\operatorname{supp} \rho \subset[-R, R]$, and consider the test functions $\rho_{\varepsilon}(z)$ of the form

$$
\rho_{\varepsilon}(z)=\rho\left(z_{1}\right)+\sqrt{\varepsilon} \rho^{\prime}\left(z_{1}\right) \chi\left(\xi / \varepsilon+z_{1} / \sqrt{\varepsilon}, z^{\prime} / \sqrt{\varepsilon}\right)
$$

with $\chi(y)$ being the 1-periodic in $y_{1}$ solution of the standard cell problem

$$
-\frac{\partial}{\partial y_{i}}\left(\theta^{*}(\xi, y) a_{i j}(\xi, y) \frac{\partial}{\partial y_{j}}\left(\chi+y_{1}\right)\right)+B(\xi, y) \cdot \nabla\left(\chi+y_{1}\right)=0
$$

in $(-\infty, \infty) \times \omega$ with the boundary condition

$$
\frac{\partial\left(\chi+y_{1}\right)}{\partial \nu_{a}}=0 \quad \text { on }(-\infty, \infty) \times \partial \omega
$$

Since $\operatorname{div}_{y} B(\xi, y)=0, B(\xi, y) \cdot \nu=0$ when $y^{\prime} \in \partial \omega$ and $\int_{(0,1) \times \omega} B_{1}(\xi, y) d y=$ $-\frac{\partial \bar{H}}{\partial p_{1}}\left(p_{1}(\xi), \xi\right)=0$, there exists a unique solution of (5.33), (5.34). Then, integrating by parts in the first term $I_{\varepsilon}^{(1)}$ of the first line of (5.32) and changing the variables $y_{1}=\xi / \varepsilon+z_{1} / \sqrt{\varepsilon}, y^{\prime}=z^{\prime} / \sqrt{\varepsilon}$, we obtain

$$
\begin{aligned}
I_{\varepsilon}^{(1)}= & \varepsilon^{n / 2} \int d y_{1} \int_{\omega} d y^{\prime} \psi_{\varepsilon}\left(B_{1} \chi-\theta^{*} a_{11}-\theta^{*} a_{1 j} \frac{\partial \chi}{\partial y_{j}}-\frac{\partial}{\partial y_{i}}\left(\theta^{*} a_{i 1} \chi\right)\right) \rho^{\prime \prime} \\
& +\varepsilon^{n / 2} \int d y_{1} \int_{\partial \omega} \psi_{\varepsilon} \theta^{*} \nu_{i} a_{i 1} \chi \rho^{\prime \prime} d S+O\left(\varepsilon^{n / 2}\right) \\
= & -\varepsilon^{n / 2} q \int \hat{\psi}\left(\sqrt{\varepsilon} y_{1}-\xi / \sqrt{\varepsilon}\right) \rho^{\prime \prime}\left(\sqrt{\varepsilon} y_{1}-\xi / \sqrt{\varepsilon}\right) d y_{1}+O\left(\varepsilon^{n / 2}\right) \\
= & -\varepsilon^{(n-1) / 2} q \int_{-R}^{R} \hat{\psi}\left(z_{1}\right) \rho^{\prime \prime}\left(z_{1}\right) d z_{1}+O\left(\varepsilon^{n / 2}\right),
\end{aligned}
$$

where the integrals with respect to $y_{1}$ in the first, second and third lines are actually taken over $(\xi / \varepsilon-R / \sqrt{\varepsilon}, \xi / \varepsilon+R / \sqrt{\varepsilon})$,

$$
\begin{aligned}
q & =\int_{(0,1) \times \omega}\left(\theta^{*} a_{11}+\theta^{*} a_{1 j} \frac{\partial \chi}{\partial y_{j}}-B_{1} \chi\right) d y \\
& =\int_{(0,1) \times \omega} \theta^{*} a_{i j} \frac{\partial}{\partial y_{i}}\left(\chi+y_{1}\right) \frac{\partial}{\partial y_{j}}\left(\chi+y_{1}\right) d y .
\end{aligned}
$$

Next we establish asymptotic formulas for other terms in (5.32). The terms in the two last lines are of order $O\left(\varepsilon^{n / 2}\right)$. The same is true for the integral $I_{\varepsilon}^{(2)}$ in the second line, though it is a little less obvious. First, we establish that

$$
I_{\varepsilon}^{(2)}=\varepsilon^{(n-1) / 2} \int_{-R}^{R} \hat{\psi}\left(z_{1}\right)\left(K_{1} z_{1} \rho^{\prime}\left(z_{1}\right)+K_{2} \rho\left(z_{1}\right)\right) d z_{1}+O\left(\varepsilon^{n / 2}\right),
$$


with

$$
\begin{aligned}
K_{1} & =\int_{(0,1) \times \omega}\left(\frac{\partial B_{1}}{\partial x_{1}}(\xi, y)+\nabla \chi \cdot \frac{\partial B}{\partial x_{1}}(\xi, y)\right) d y \\
K_{2} & =\int_{(0,1) \times \omega} \frac{\partial B_{1}}{\partial x_{1}}(\xi, y) d y .
\end{aligned}
$$

Then we observe that

$$
\int_{(0,1) \times \omega} B_{1}\left(x_{1}, y\right) d y=-\frac{\partial \bar{H}}{\partial p_{1}}\left(p_{1}\left(x_{1}\right), x_{1}\right)
$$

therefore

$$
K_{2}=-\left.\frac{\partial}{\partial x_{1}} \frac{\partial \bar{H}}{\partial p_{1}}\left(x_{1}, p_{1}\left(x_{1}\right)\right)\right|_{x_{1}=\xi}=0
$$

because of conditions (5.1)-(5.4). We also know that $\operatorname{div}_{y} B\left(x_{1}, y\right)=0$ and $B\left(x_{1}, y\right) \nu=0$ when $y^{\prime} \in \partial \omega$, hence $K_{1}=0$. Finally, the asymptotic formulas for the sum $I_{\varepsilon}^{(3)}$ of the terms in the third line of (5.32) and the second term $\tilde{I}_{\varepsilon}^{(1)}$ in the first line are:

$$
\begin{aligned}
I_{\varepsilon}^{(3)}= & \varepsilon^{(n-1) / 2} \mu_{\varepsilon} \int_{-R}^{R} \psi\left(z_{1}\right) \rho\left(z_{1}\right) d z_{1}+\left(1+\left|\mu_{\varepsilon}\right|\right) O\left(\varepsilon^{n / 2}\right) \\
& -\left.\varepsilon^{(n-1) / 2} \int_{-R}^{R} d z_{1} \psi\left(z_{1}\right) \rho\left(z_{1}\right) \int_{(0,1) \times \partial \omega} \theta^{*} \nu_{i} a_{i 1} \frac{\partial \log \theta}{\partial x_{1}}\right|_{x_{1}=\xi} d S \\
\tilde{I}_{\varepsilon}^{(1)}= & \varepsilon^{(n-1) / 2} \int_{-R}^{R} \frac{V}{2} z_{1}^{2} \hat{\psi}\left(z_{1}\right) \rho\left(z_{1}\right) d z_{1}+O\left(\varepsilon^{n / 2}\right) \\
& +\varepsilon^{(n-1) / 2} \int_{-R}^{R} d z_{1} \hat{\psi}\left(z_{1}\right) \rho\left(z_{1}\right) \\
& \quad \times\left.\int_{(0,1) \times \omega} \frac{\theta^{*}}{\theta}\left(a_{11}\left(p_{1}^{\prime} \theta+\left(2 p_{1}-b_{1}\right) \frac{\partial \theta}{\partial x_{1}}\right)-2 a_{1 j} \frac{\partial^{2} \theta}{\partial x_{1} \partial y_{j}}\right)\right|_{x_{1}=\xi} d y,
\end{aligned}
$$

where $\theta^{*}=\theta^{*}\left(x_{1}, y\right), \theta=\theta\left(y, x_{1}, p_{1}\left(x_{1}\right)\right), p_{1}=p_{1}\left(x_{1}\right), a_{i j}=a_{i j}\left(x_{1}, y\right), b_{j}=$ $b_{j}\left(x_{1}, y\right)$. Thus, substituting the above obtained asymptotic formulas for the terms in (5.32) and dividing by $\varepsilon^{(n-1) / 2}$, we get

$$
\begin{aligned}
\int_{\mathbb{R}} \hat{\psi}\left(z_{1}\right)\left(-q \rho^{\prime \prime}\left(z_{1}\right)+\frac{1}{2} V z_{1}^{2} \rho\left(z_{1}\right)+m \rho\left(z_{1}\right)-\mu_{\varepsilon} \rho\left(z_{1}\right)\right) d z_{1} & \\
= & \left(1+\left|\mu_{\varepsilon}\right|\right) O(\sqrt{\varepsilon}),
\end{aligned}
$$

where

$$
\begin{aligned}
m=\int_{(0,1) \times \omega} \frac{\theta^{*}}{\theta}\left(a _ { 1 1 } \left(p_{1}^{\prime} \theta+\left(2 p_{1}-\right.\right.\right. & \left.\left.\left.b_{1}\right) \frac{\partial \theta}{\partial x_{1}}\right)-2 a_{1 j} \frac{\partial^{2} \theta}{\partial x_{1} \partial y_{j}}\right)\left.\right|_{x_{1}=\xi} d y \\
& +\left.\int_{(0,1) \times \partial \omega} \theta^{*} \nu_{i} a_{i 1} \frac{\partial \log \theta}{\partial x_{1}}\right|_{x_{1}=\xi} d S .
\end{aligned}
$$


Since $\rho$ is an arbitrary function (smooth and compactly supported), we conclude that $\left|\mu_{\varepsilon}\right|$ is bounded; otherwise, $\hat{\psi}$ is identically zero that contradicts (5.31). Now, passing to the limit in (5.37), we arrive at the weak formulation of (5.28).

Remark 5.2. The eigenfunction $\theta^{*}$ is represented as the product $\theta^{*}=\theta \vartheta$ of the eigenfunction $\theta$ of problem (3.4), (3.5) and the eigenfunction $\vartheta$ of the problem adjoint to (3.4), (3.5).

Remark 5.3. Definition (5.38) of $m$ is actually independent of any particular choice of the function $p_{1}\left(x_{1}\right): p_{1}(\xi)$ is uniquely determined as the minimizer of $\bar{H}\left(p_{1}, \xi\right)$, and under conditions (5.1)-(5.4),

$$
p^{\prime}(\xi)=-\frac{\partial^{2} \bar{H}}{\partial p_{1} \partial x_{1}}\left(\xi, p_{1}(\xi)\right) / \frac{\partial^{2} \bar{H}}{\partial p_{1}^{2}}\left(\xi, p_{1}(\xi)\right)
$$

Now consider the auxiliary problem

$$
\hat{\mathcal{L}}_{\varepsilon} \psi:=-q \Delta \psi+\frac{1}{2} V z_{1}^{2} \psi+(m+\Lambda) \psi=\theta^{*} f \quad \text { in } \Omega_{\sqrt{\varepsilon}}
$$

with the Neumann condition $\frac{\partial \psi}{\partial \nu}=0$ on $\partial \Omega_{\sqrt{\varepsilon}}$. In the operator form it reads $\frac{1}{\theta^{*}} \hat{\mathcal{L}}_{\varepsilon} \psi=f$. We endow $L^{2}\left(\Omega_{\sqrt{\varepsilon}}\right)$ with the norm $\|\psi\|_{\varepsilon, \theta^{*}}^{2}=\frac{1}{|\sqrt{\varepsilon} \omega|} \int_{\Omega_{\sqrt{\varepsilon}}} \psi^{2} \theta^{*} d z$ and the corresponding inner product, then $\frac{1}{\theta^{*}} \hat{\mathcal{L}}_{\varepsilon}$ is a selfadjoint operator. It has a discrete spectrum. Moreover, using the Courant minimax principle, one can prove that the eigenvalues of $\frac{1}{\theta^{*}} \hat{\mathcal{L}}_{\varepsilon}$ converge to the numbers $\Lambda+\hat{\mu}^{(k)}$, where $\hat{\mu}^{(1)}<$ $\hat{\mu}^{(2)}<\ldots$ are eigenvalues of $(5.28)$.

Next we show that $\left\|\left(\frac{1}{\theta^{*}} \tilde{\mathcal{L}}_{\varepsilon}\right)^{-1}-\left(\frac{1}{\theta^{*}} \hat{\mathcal{L}}_{\varepsilon}\right)^{-1}\right\| \rightarrow 0$, where $\|\cdot\|$ denotes the operator norm (over $L^{2}\left(\Omega_{\sqrt{\varepsilon}}\right)$ ). To this end, consider for a given $f_{\varepsilon} \in L^{2}\left(\Omega_{\sqrt{\varepsilon}}\right)$ with $\frac{1}{|\sqrt{\varepsilon} \omega|} \int_{\Omega_{\sqrt{\varepsilon}}} f_{\varepsilon}^{2} d z=1$ the solutions $\psi_{\varepsilon}$ and $\hat{\psi}_{\varepsilon}$ of the equations $\tilde{\mathcal{L}}_{\varepsilon} \psi_{\varepsilon}=\theta^{*} f_{\varepsilon}$ and $\hat{\mathcal{L}}_{\varepsilon} \hat{\psi}_{\varepsilon}=\theta^{*} f_{\varepsilon}$. Revising the proof of Lemma 5.1 , we see that it can be used (with minimal modifications) to show that up to a subsequence $\frac{1}{|\sqrt{\varepsilon} \omega|} \int_{\Omega_{\sqrt{\varepsilon}}}\left(\psi_{\varepsilon}^{2}-\right.$ $\psi)^{2} d z \rightarrow 0$. Here a function $\psi=\psi\left(z_{1}\right)$ solves

$$
-q \psi^{\prime \prime}\left(z_{1}\right)+\frac{1}{2} V z_{1}^{2} \psi\left(z_{1}\right)+(\Lambda+m) \psi\left(z_{1}\right)=f^{*}\left(z_{1}\right),
$$

where $f^{*}\left(z_{1}\right)$ is a weak limit of the functions

$$
\left\langle\theta^{*} f_{\varepsilon}\right\rangle\left(z_{1}\right)=\frac{1}{|\sqrt{\varepsilon} \omega|} \int_{\left\{z_{1}\right\} \times \sqrt{\varepsilon} \omega} f \tilde{\theta}^{*} d z^{\prime}
$$

(extended by 0 on $\mathbb{R} \backslash[-\xi / \sqrt{\varepsilon},(L-\xi) / \sqrt{\varepsilon}]$ ) in $L^{2}(\mathbb{R})$. The same result holds for $\hat{\psi}_{\varepsilon}$, thus $\left\|\left(\frac{1}{\theta^{*}} \tilde{\mathcal{L}}_{\varepsilon}-\lambda I\right)^{-1}-\left(\frac{1}{\theta^{*}} \hat{\mathcal{L}}_{\varepsilon}-\lambda I\right)^{-1}\right\| \rightarrow 0$ for $\lambda=0$. This result extends to the uniform in $\lambda$ resolvent convergence in operator norm on an arbitrary compact subset of $\mathbb{C} \backslash\left\{\hat{\mu}^{(1)}, \hat{\mu}^{(2)}, \ldots\right\}$, the latter in turn yields the convergence of spectral projectors in the operator norm. Thus we have proved the following result. 
Theorem 5.4. Assume that condition (5.1) is satisfied along with assumptions (2.5), (2.6). Let the eigenvalues $\lambda=\lambda_{\varepsilon}^{(k)}$ of problem (2.4) be enumerated by the magnitude of their real parts (in the decreasing order), then

$$
\lambda_{\varepsilon}^{(k)}=\bar{\lambda}-\varepsilon \hat{\mu}^{(k)}+o(\varepsilon),
$$

where $\hat{\mu}^{(k)}$ are eigenvalues of the operator $-q \frac{d^{2}}{d z_{1}^{2}}+\frac{1}{2} V z_{1}^{2}+m$ on $\mathbb{R}$, enumerated by their magnitude.

Supports. An essential part of this work was done during the visit of the second author at UiT, campus Narvik, in the autumn of 2018. The visit was supported by BFS/TFS project "Pure Mathematics in Norway".

\section{References}

[1] G. Allaire, Y. Capdeboscq, and M. Puel, Homogenization of a one-dimensional spectral problem for a singularly perturbed elliptic operator with Neumann boundary conditions, Discrete Contin. Dyn. Syst. Ser. B 17 (2012), No. 1, 1-31.

[2] G. Allaire and A. Piatnitski, On the asymptotic behaviour of the kernel of an adjoint convection-diffusion operator in a long cylinder, Rev. Mat. Iberoam. 33 (2017), No. 4, 1123-1148.

[3] M. Arisawa, Long time averaged reflection force and homogenization of oscillating Neumann boundary conditions, Ann. Inst. H. Poincaré (C) Anal. Non Linéaire, 20 (2003), No. 2, 293-332.

[4] G. Barles, F. Da Lio, P.-L. Lions, and P.E. Souganidis, Ergodic problems and periodic homogenization for fully nonlinear equations in half-space type domains with Neumann boundary conditions, Indiana Univ. Math. J. 57 (2008), No. 5, 2355-2375.

[5] I. Capuzzo-Dolcetta and P.-L. Lions, Hamilton-Jacobi equations with state constraints, Trans. Amer. Math. Soc. 318 (1990), 643-683.

[6] D. Gilbarg and N.S. Trudinger, Elliptic Partial Differential Equations of Second Order, Springer-Verlag, Berlin-Heidelberg-New York-Tokyo, 1983.

[7] M.G. Crandall, H. Ishii, and P.-L. Lions, User's guide to viscosity solutions of second order partial dierential equations, Bull. Amer. Math. Soc. (N.S.) 27 (1992), 1-67.

[8] L.C. Evans, The perturbed test function method for viscosity solutions of nonlinear PDE, Proc. Roy. Soc. Edinburgh Sect. A 111 (1989), No. 3-4, 359-375.

[9] G.M. Lieberman, Oblique derivative problems in Lipschitz domains. II. Discontinuous boundary data, J. Reine Angew. Math. 389 (1988), 1-21.

[10] I. Pankratova and A. Piatnitski, On behavior at infinity of solutions to stationary convection-diffusion equation in a cylinder, Discrete Contin. Dyn. Syst. Ser. B 11 (2009), No. 4, 935-970.

[11] A. Piatnitski and V. Rybalko, On the first eigenpair of singularly perturbed operators with oscillating coefficients, Comm. Partial Differential Equations 41 (2016), No. 1, 1-31. 
[12] A. Piatnitski, A. Rybalko, and V. Rybalko, Ground states of singularly perturbed convection-diffusion equation with oscillating coefficients, ESAIM Control Optim. Calc. Var. 20 (2014), No. 4, 1059-1077.

[13] A. Piatnitski, A. Rybalko, and V. Rybalko, Singularly perturbed spectral problems with Neumann boundary conditions, Complex Var. Elliptic Equ. 61 (2015), No. 2, $252-274$.

[14] H.M. Soner, Optimal control with state-space constraint. I., SIAM J. Control Optim. 24 (1986), 552-561.

Received April 1, 2019.

Andrey Piatnitski,

The Arctic University of Norway, Campus in Narvik, P.O. Box 385, N-8505 Narvik, Norway,

Institute for Information Transmission Problems RAS, Bolshoi Karetnyi, 19, Moscow, 127051, Russia,

E-mail: apiatnitski@gmail.com

Volodymyr Rybalko,

B. Verkin Institute for Low Temperature Physics and Engineering of the National Academy of Sciences of Ukraine, 47 Nauky Ave., Kharkiv, 61103, Ukraine,

E-mail: vrybalko@ilt.kharkov.ua

\section{Сингулярно збурені спектральні проблеми в тонкому циліндрі з умовами Фур'є на його основах}

Andrey Piatnitski and Volodymyr Rybalko

У роботі вивчається нижня частина спектра сингулярно збуреного еліптичного оператора другого порядку, який визначено в тонкому циліндрі та який має періодичні коефіцієнти в подовжньому напрямі. Розглянуто однорідну умову Неймана на бічній поверхні циліндра та однорідну умову Фур'є загального вигляду на його основах. Доведено, що асимптотичне поводження першої власної пари може бути охарактеризовано в термінах граничної одновимірної проблеми для ефективного рівняння Гамільтона-Якобі з ефективними крайовими умовами. Для того щоб побудувати коректори примежового шару, вивчається спектральна проблема типу Стеклова в напівнескінченному циліндрі (ці результати мають окремий інтерес). За структурних припущень відносно ефективної проблеми, які ведуть до локалізації (у деякому сенсі) власних функцій всередині циліндра, доведено двочленну асимптотичну формулу для першого та наступних власних значень.

Ключові слова: сингулярно збурені оператори, задача усереднення, власні значення, власні функції, крайова умова Фур'є. 